\title{
CONTRACTIVE PROJECTIONS IN CONTINUOUS FUNCTION SPACES
}

\author{
KARL LINDBERG ${ }^{1}$
}

\begin{abstract}
Let $C(K)$ be the Banach space of real-valued continuous functions on a compact Hausdorff space with the supremum norm and let $X$ be a closed subspace of $C(K)$ which separates points of $K$. Necessary and sufficient conditions are given for $X$ to be the range of a projection of norm one in $C(K)$. It is shown that the form of a projection of norm one is determined by a real-valued continuous function which is defined on a subset of $K$ and which satisfies conditions imposed by $X$. When there is a projection of norm one onto $X$, it is shown that there is a one-to-one correspondence between the continuous functions which satisfy the conditions imposed by $X$ and the projections of norm one onto $X$.
\end{abstract}

Using well-known results of Nachbin, Goodner and Kelley (see [2, p. 2]), it is easily demonstrated that if $S$ is an extremely disconnected compact Hausdorff space, then $X$, a closed subspace of $C(S)$, is the range of a norm one projection in $C(S)$ iff $X$ itself is isometric to the continuous functions on an extremely disconnected compact Hausdorff space. The main purpose of this research is to give necessary and sufficient conditions for a closed separating subspace $X$ of $C(K), K$ a compact Hausdorff space, to be the range of a norm one projection in $C(K)$. Theorem 3.1 supplies these conditions. Proposition 1.2 shows that the form of each contractive projection onto $X$ can be given in terms of a real-valued continuous function, determined by the projection, which is defined on a subset of $K$. This continuous function satisfies certain conditions which depend only on the subspace $X$. Theorem 3.1 also shows that when there is a contractive projection onto $X$ there is a one-to-one correspondence between contractive projections onto $X$ and the continuous functions which satisfy these conditions imposed by $X$. We give another proof to the fact, proved in [3], that the Banach space $Y$ is isometric to the range of a contractive

Received by the editors June 11, 1971 and, in revised form, February 9, 1972.

AMS 1970 subject classifications. Primary 46E15, 47B99.

Key words and phrases. Contractive projection, extreme points, involutive homeomorphism, isometry.

${ }^{1}$ The results of this paper were obtained while the author was working on his Ph.D. thesis prepared at the University of California, Berkeley, under the supervision of Professor H. P. Rosenthal. 
projection in some space $C(Q), Q$ compact Hausdorff, iff $Y$ is isometric to $C_{\sigma}(L)$ for some compact Hausdorff space $L$ and involutive homeomorphism $\sigma$ of $L$. We also extend some results of [4].

Throughout this paper $K$ is to be a compact Hausdorff space and $C(K)$ is to be the Banach space of real-valued continuous functions on $K$ endowed with the supremum norm. A projection in a Banach space $Y$ is a bounded linear operator $P$ on $Y$ such that $P^{2}=P$; a contractive projection is a projection of norm one. Subspace is to mean closed subspace.

A subspace $Y$ of $C(K)$ is said to be separating if, for each $x \neq y$ in $K$, there is an $f$ in $Y$ such that $f(x) \neq f(y)$. An involutive homeomorphism $T$ of a topological space is a homeomorphism such that $T^{2}$ is the identity map. If $\delta$ is an involutive homeomorphism of $K$, then $C_{\delta}(K)$ is the space $\{f$ in $C(K): f(x)=-f(\delta(x))$ for all $x$ in $K$ \} endowed with the supremum norm. By an isometry $T$ from the Banach space $Y$ onto the Banach space $Z$ we mean a bounded linear map with bounded inverse such that $\|T(f)\|_{Z}=$ $\|f\|_{Y}$ for all $f$ in $Y$; in this case $Y$ and $Z$ are said to be isometric. If $Y$ is a Banach space, $B_{Y}$, the unit ball of $Y$, is $\{y$ in $Y:\|y\| \leqq 1\}$. If $x$ is in $K, \pm x^{\prime}$ is the linear functional in $C(K)^{*}$ such that $\pm x^{\prime}(f)= \pm f(x)$. It is shown in $[1$, p. 441] that for any closed subspace $Y$ of $C(K)$, the extreme points of $B_{Y}$ *are of the form $\pm\left. x^{\prime}\right|_{Y}$. A point $x$ in $K$ is a double point of $X$, a separating subspace, if there is a $y$ in $K$ such that for all $f$ in $X, f(x)=-f(y)$. A point of $K$ is a single point if it is not a double point. By single (respectively double) extreme point we mean a single (respectively double) point $x$ such that $\left.x^{\prime}\right|_{X}$ is an extreme point of $B_{X^{*}}$. The double points are closed and the map $\bar{\sigma}$, defined on the double points by $\bar{\sigma}(x)=y$ where $f(y)=-f(x)$ for all $f$ in $X$, is an involutive homeomorphism of the double points. Now let $S$ be the closure in $K$ of the single extreme points; let $D$ be the closure of the double extreme points and let $\sigma$ be $\left.\bar{\sigma}\right|_{D}$. The map $\sigma$ is an involutive homeomorphism of $D$ since $\bar{\sigma}$ maps double extreme points into double extreme points.

Throughout this paper $X$ is to denote a separating subspace of $C(K)$ and $S, D$ and $\sigma$ are to be as stated above.

\section{Necessary conditions for the existence of a contractive projection.}

Proposition 1.1. Let $P$ be a contractive projection in $C(K)$ onto $X$. For all $x$ in $S, P^{*}\left(\left.x^{\prime}\right|_{x}\right)=x^{\prime}$; for all $x$ in $D$, there is a $t, 0 \leqq t \leqq 1$, such that

$$
P^{*}\left(\left.x^{\prime}\right|_{x}\right)=t x^{\prime}-(1-t)(\sigma(x))^{\prime} .
$$

Proof. Let $x$ be in $K$ such that $\left.x^{\prime}\right|_{X}$ is an extreme point of $B_{X^{*}}$. Let $E_{x}=\left\{x^{*}\right.$ in $\left.\left.B_{C\left(K^{*}\right)^{*}: x^{*}}\right|_{x}=\left.x^{\prime}\right|_{x^{\prime}}\right\} . E_{x}$ is convex and $w^{*}$-closed since $E_{x}=$ $\bigcap_{f \in X}\left\{x^{*}\right.$ in $\left.B_{C(K)^{*}}: x^{*}(f)=f(x)\right\}$. Hence $E_{x}$ is the closed convex hull of its extreme points. If $x *$ is an extreme point of $E_{x}$, it is an extreme point of 
$B_{C(K)^{*}}$. This is true since if $x^{*}=\frac{1}{2} y^{*}+\frac{1}{2} z^{*}$ where $y^{*}$ and $z^{*}$ are in $B_{C(K)^{*}}$, then $\left.x^{\prime}\right|_{X}=\left.x^{*}\right|_{X^{*}}=\left.\frac{1}{2} y^{*}\right|_{X}+\left.\frac{1}{2} z^{*}\right|_{X}$. Since $\left.x^{\prime}\right|_{X}$ is an extreme point of $B_{X^{*}}$, $\left.y^{*}\right|_{x}=\left.z^{*}\right|_{x}=\left.x^{*}\right|_{x}=\left.x^{\prime}\right|_{X}$ and hence $y^{*}$ and $z^{*}$ are in $E_{x}$. But since $x^{*}$ is an extreme point of $E_{x}$, we have $x^{*}=y^{*}=z^{*}$ and $x^{*}$ is an extreme point of $B_{C(K)^{*}}$. It follows that $x^{*}= \pm y^{\prime}$ for some $y$ in $K$. Now using the fact that $X$ separates points and $\left.x^{*}\right|_{X}=x^{\prime}$, we have that if $x^{*}=y^{\prime}$, then $x=y$ and if $x^{*}=-y^{\prime}, y=\sigma(x)$. Hence if $x$ is a single extreme point, $E_{x}=\left\{x^{\prime}\right\}$; and if $x$ is a double extreme point, $E_{x}$ is the closed convex hull of $x^{\prime}$ and $-y^{\prime}=-(\sigma(x))^{\prime}$, in which case $E_{x}$ consists of exactly those $y^{*}$ such that $y^{*}=t x^{\prime}+(1-t)\left(-(\sigma(x))^{\prime}\right)=t x^{\prime}-(1-t)(\sigma(x))^{\prime}$ for some $t, 0 \leqq t \leqq 1$. Now, $P^{*}\left(\left.x^{\prime}\right|_{X}\right)$ is in $E_{x}$. Therefore, if $x$ is a single extreme point, $P^{*}\left(\left.x^{\prime}\right|_{X}\right)=x^{\prime}$; and if $x$ is a double extreme point, there is a $t, 0 \leqq t \leqq 1$, such that $P^{*}\left(\left.x^{\prime}\right|_{x}\right)=t x^{\prime}-(1-t)(\sigma(x))^{\prime}$. The conclusion of the proposition now follows by noticing that if $\left\{x_{\alpha}\right\}$ is a net converging to $x$ in $K$, then $P^{*}\left(\left.x_{\alpha}^{\prime}\right|_{x}\right)$ converges $x^{*}$ to $P^{*}\left(\left.x^{\prime}\right|_{X}\right)$.

PROPOSITION 1.2. If there is a contractive projection $P$ in $C(K)$ onto $X$, then $S \cap \sigma(S \cap D)=\varnothing$ and there is a continuous function $c$ from $S \cup D$ into $[0,1]$ (the unit interval), uniquely determined by $P$, such that for all $f$ in $C(K)$ :

and

$$
P(f)(x)=c(x) f(x)=f(x) \text { for } x \text { in } S
$$

$$
P(f)(x)=c(x) f(x)-(1-c(x)) f(\sigma(x)) \text { for } x \text { in } D .
$$

Furthermore, $c(x)=1$ for $x$ in $S$ and $c(x)+c(\sigma(x))=1$ for $x$ in $D$.

Proof. For all $x$ in $S \cup D$, define $c(x)=\frac{1}{2}\left(P\left(1_{K}\right)(x)+1\right)$, where $1_{K}$ is the function identically one on $K$. Since $P$ is a contractive projection, $-1 \leqq P\left(1_{K}\right)(x) \leqq 1$; therefore $c$ has the desired range. By Proposition 1.1, for $x$ in $S, P(f)(x)=f(x)$ for all $f$ in $C(K)$ and hence $P\left(1_{K}\right)(x)=1$ and $c(x)=1$. Also by Proposition 1.1 , for $x$ in $D$, there is a $t$ such that $P^{*}\left(\left.x^{\prime}\right|_{\mathrm{x}}\right)=t x^{\prime}-(1-t)(\sigma(x))^{\prime}$. Hence, $P\left(1_{K}\right)(x)=2 t-1$ and $c(x)=t$. Therefore for all $f$ in $C(K)$ and $x$ in $D, P(f)(x)=c(x) f(x)-(1-c(x)) f(\sigma(x))$. Since for all $f$ in $X, f(x)=-f(\sigma(x))$, we have that for $x$ in $D, c(x)+$ $c(\sigma(x))=\frac{1}{2}\left[P\left(1_{K}\right)(x)+1+P\left(1_{K}\right)(\sigma(x))+1\right]=1$. This property of $c$ along with the property that for $x$ in $S, c(x)=1$, yields that $S \cap \sigma(S \cap D)=\varnothing$.

Proposition 1.3. If there is a contractive projection $P$ in $C(K)$ onto $X$, then $X$ is isometric by restriction to $S \cup D$ to a subspace $Y$ of $C(S \cup D)$ where $Y=\{f$ in $C(S \cup D): f(x)=-f(\sigma(x))$ for $x$ in $D\}$.

Proof. The restriction map is linear. It preserves norm since for $f$ in $X$, $\|f\|_{X}=\sup _{x^{*} \in \operatorname{Ext} B_{X^{*}}}\left|x^{*}(f)\right|$; but since every extreme point of $B_{X^{*}}$ is of the form $\pm\left. x^{\prime}\right|_{X}$ for some $x$ in $S \cup D$, we have $\|f\|_{X}=\sup _{x \in S \cup D}|f(x)|=$ $\left\|\left.f\right|_{S \cup D}\right\|_{C(S \cup D)}$. We need only show this restriction map is onto $Y$. Given 
$f$ in $Y$ choose $\bar{f}$ in $C(K)$ such that $\left.\bar{f}\right|_{S \cup D}=f$. Now $P(\bar{f})$ is in $X$ and $\left.P(\bar{f})\right|_{S \cup D}=f$ since, by Proposition 1.2, if $x$ is in $S, P(\bar{f})(x)=\bar{f}(x)=f(x)$ and if $x$ is in $D, P(\bar{f})(x)=c(x) \bar{f}(x)-(1-c(x)) \bar{f}(\sigma(x))=f(x)$.

Corollary 1.4. Assume the hypotheses of Proposition 1.3.

(a) If for all $x$ in $D, P\left(1_{K}\right)(x) \neq 1$, then $X$ is isometric by restriction to $S \cup D$ to $C(S) \oplus C_{\sigma}(D)$ with the supremum norm sum. If $\|I-P\|<2$ where $I$ is the identity operator on $C(K)$, then $P\left(1_{K}\right) \neq 1$ for $x$ in $D$.

(b) If $P\left(1_{K}\right) \neq 0$ for all $x$ in $D$, then there is a closed subset $Q$ of $K$ such that $X$ is isometric by restriction to $Q$ to $C(Q)$.

Proof. (a) If $P\left(1_{K}\right) \neq 1$ for all $x$ in $D$, by Proposition 1.2, $c(x) \neq 1$ for all $x$ in $D$ and $S \cap D=\varnothing$. Now we may use Proposition 1.3.

(b) If $P\left(1_{K}\right)(x) \neq 0$, then $c(x) \neq \frac{1}{2}$ for $x$ in $D$ and, using Proposition 1.2, we have $D$ is the disjoint union of two closed homeomorphic sets, $A$ and $\sigma(A)$, where $A=\left\{x\right.$ in $\left.D: c(x)>\frac{1}{2}\right\}$. Let $Q=S \cup A$.

COROLlaRY 1.5. Let $Y$ be any closed subspace of $C(K)$. If there is a contractive projection $P$ in $C(K)$ onto $Y$, then $Y$ is isometric to $C_{\delta}(L)$ where $L$ is a compact Hausdorff space and $\delta$ an involutive homeomorphism of $L$.

Proof. We may assume $Y$ is a separating subspace of $C(K)$ since $Y$ is isometric to a separating subspace of $C(Q)$ where $Q$ is the quotient space of $K$ obtained by equating points in $K$ on which all functions in $Y$ agree. $C(Q)$ may be considered as a subspace of $C(K)$ and hence $\left.P\right|_{C(Q)}$ is a contractive projection in $C(Q)$ onto $Y$. Therefore, by Proposition 1.3, there are sets $S, D$ and an involutive homeomorphism $\sigma$ of $D$ such that $S \cap$ $\sigma(S \cap D)=\varnothing$ and $Y$ is isometric to $\{f$ in $C(S \cup D): f(x)=-f(\sigma(x))$ for all $x$ in $D$ \}. We now show this space is isometric to $C_{\delta}(L)$. Let $L$ be the set of points consisting of $S \cup D$ together with the set $A$ which is a copy of $S-D$, where a point $x$ in $S-D$ is identified with $\bar{x}$ in $A$. Now define $\delta$ as follows: $\delta(x)=\sigma(x)$ for $x$ in $D, \delta(x)=\bar{x}$ for $x$ in $S-D$, and $\delta(\bar{x})=x$ for $\bar{x}$ in $A$. A basis for the topology on $L$ is as follows: An open neighborhood of a point $x$ in $S \cup D-\sigma(S \cap D)$ is of the form $N_{x}-\sigma(S \cap D)$ where $N_{x}$ is an open neighborhood of $x$ in $S \cup D$; an open neighborhood of a point $y$ in $\sigma(S \cap D) \cup A$ is of the form $\delta\left(N_{x}\right)$ where $\delta(x)=y$ and $N_{x}$ is an open neighborhood of $x$ in $S \cup D$. With this topology $L$ is a compact Hausdorff space and $\delta$ is an involutive homeomorphism of $L$ such that $\left.\delta\right|_{D}=\sigma$. Now the map extending each function $f$ in $\{f$ in $C(S \cup D): f(\sigma(x))=-f(x)\}$ to $\bar{f}$ in $C_{\delta}(L)$ by $\bar{f}(\bar{x})=-f(\delta(\bar{x}))$ is an isometry onto $C_{\delta}(L)$. Hence $Y$ is isometric to $C_{\delta}(L)$.

EXAMPLE 1.6. Consider $C[0,1]$, the continuous real-valued functions on the unit interval. Let $Y=\{f$ in $C[0,1]: f(0)=-f(1)\}$. The single extreme points of $Y$ are the points of the open interval $(0,1)$; the double 
extreme points are the points $\{0,1\}$. Hence $D=\{0,1\}$ and $S=[0,1]$. The involutive homeomorphism of $D$ is $\sigma(0)=1$. Now, since $S \cap \sigma(S \cap D)=$ $D \neq \varnothing$, there is no contractive projection in $C[0,1]$ onto $Y$ by Proposition 1.2 .

REMARKS. If $\delta$ is any involutive homeomorphism of $K$ we observe that $C_{\delta}(K)$ is the range of the contractive projection $P$ in $C(K)$ where $P(f)(x)=$ $\frac{1}{2} f(x)-\frac{1}{2} f(\delta(x))$ for all $f$ in $C(K)$ and all $x$ in $K$. This observation along with Corollary 1.5 gives another proof of the following result of [3]: A Banach space $Y$ is isometric to the range of a contractive projection in some $C(Q)$ iff $Y$ is isometric to some $C_{\delta}(L)$. This result does not help us determine those subspaces of $C(K)$ which are the range of a contractive projection in $C(K)$. Example 1.6 gives a subspace $Y$ which, although not the range of a contractive projection in $C[0,1]$, is isometric to $C_{\sigma}\left(S_{1}\right)$ where $S_{1}$ is the unit circle and $\sigma$ is the antipodal map.

In [4] it is shown that a subspace $Y$ of $C(K)$ is isometric to $C(L), L$ a compact Hausdorff space, if $Y$ is the range of a contractive projection in $C(K)$ and $Y$ has the following property: $Y$, considered as a separating subspace of $C(Q)$, where $Q$ is the quotient space of $K$ obtained by equating points of $K$ on which all functions in $Y$ agree, has no double extreme points. This result follows from Corollary 1.4 since $Y$ can be considered as the range of a contractive projection in $C(Q)$ with $D=\varnothing$.

\section{Sufficient conditions for the existence of a contractive projection.}

Proposition 2.1. There is a continuous function from $S \cup D$ into $[0,1]$ such that $c(x)+c(\sigma(x))=1$ for $x$ in $D$ and $c(x)=1$ for $x$ in $S$ iff $S \cap \sigma(S \cap D)=$ $\varnothing$.

Proof. If such a $c$ exists, $c(\sigma(x))=0$ for all $x$ in $S \cap D$; hence $S \cap$ $\sigma(S \cap D)=\varnothing$. Suppose $S \cap \sigma(S \cap D)=\varnothing$. If $S \cap D=\varnothing, c(x)=\frac{1}{2}$ for $x$ in $D$ and $c(x)=1$ for $x$ in $S$ has the desired properties; so assume $S \cap D \neq \varnothing$. There are open, disjoint sets $u$ and $v$ in $D$ with the relative topology such that $S \cap D$ is contained in $u$ and $\sigma(S \cap D)$ is contained in $v$. For each $x$ in $S \cap D$, there is an open set $u_{x}$ containing $x$ and contained in $u$ with $\sigma\left(u_{x}\right)$ contained in $v$. Let $u^{\prime}=\bigcup_{x \in S \cap D} u_{x}$ and $v^{\prime}=\sigma\left(u^{\prime}\right)$. Now $u^{\prime}$ and $v^{\prime}$ are disjoint open sets in $D$. Choose a continuous function $d$ from $D$ into $[0,1]$ such that $\left.d\right|_{\sigma(S \cap D)}=0$ and $d(x)=1$ for $x$ not in $v^{\prime}$. We have $d(x)=1$ for $x$ in $u^{\prime}$ and $d(x)+d(\sigma(x)) \neq 0$ for $x$ in $D$. Define $c(x)=d(x) /(d(x)+d(\sigma(x)))$ for all $x$ in $D$ and $c(x)=1$ for $x$ in $S$. Now $c$ has the desired properties.

Proposition 2.2. If $S \cap \sigma(S \cap D)=\varnothing$ and $X$ is isometric by restriction to $S \cup D$ to $Y=\{f$ in $c(S \cup D): f(x)=-f(\sigma(x))$ for $x$ in $D\}$, then there is $a$ contractive projection in $C(K)$ onto $X$. 
Proof. Let $R$ be the restriction isometry from $X$ onto $Y$. By Proposition 2.1 there is a continuous function $c$ from $S \cup D$ into $[0,1]$ such that $c(x)=1$ for $x$ in $S$ and $c(x)+c(\sigma(x))=1$ for $x$ in $D$. Define a map $T_{c}$ from $C(K)$ onto $Y$ such that for $f$ in $C(K), T_{c} f(x)=f(x)$ for $x$ in $S$ and $T_{c}(f)(x)=c(x) f(x)-(1-c(x)) f(\sigma(x))$ for $x$ in $D$. For each $f$ in $C(K)$, $T_{c}(f)$ is continuous on $S \cup D$ since $c$ is continuous; also for $x$ in $D$,

$$
\begin{aligned}
T_{c}(f)(\sigma(x)) & =c(\sigma(x)) f(\sigma(x))-(1-c(\sigma(x))) f\left(\sigma^{2} x\right) \\
& =(1-c(x)) f(\sigma(x))-c(x) f(x)=-T_{c}(f)(x) .
\end{aligned}
$$

Hence $T_{c}$ is a map into $Y ; T_{c}$ is linear. We have $\left\|T_{c}\right\|=1$ since for $f$ in $X$, $T_{c}(f)=\left.f\right|_{S \cup D}=R(f)$, and for all $f$ in $C(K),\left|T_{c}(f)(x)\right|=|f(x)|$ for $x$ in $S$, and for $x$ in $D,\left|T_{c}(f)(x)\right| \leqq|c(x)+(1-c(x))|\|f\|=\|f\|$. Also $T_{c}$ is onto $Y$ since for $f$ in $Y, R^{-1}(f)$ is in $X$ and $T_{c}\left(R^{-1}(f)\right)=R\left(R^{-1}(f)\right)=f$. Now define $P_{c}=R^{-1} T_{c} . P_{c}$ is a linear operator in $C(K)$ with range $X$ and $\left\|P_{c}\right\| \leqq$ $\left\|R^{-1}\right\| \cdot\left\|T_{c}\right\|=1$. For $f$ in $X, P_{c}(f)=R^{-1} T_{c}(f)=R^{-1}(R(f))=f$. Hence $P_{c}$ is a contractive projection $C(K)$ onto $X$.

\section{The main theorem.}

THEOREM 3.1. Let $X$ be a closed separating subspace of $C(K)$. Let $S$ (respectively $D$ ) be the closure in $K$ of the single (respectively double) extreme points of $X$. Let $\sigma$ be the involutive homeomorphism of $D$ such that for all $f$ in $X, f(\sigma(x))=-f(x)$ for all $x$ in $D$. There is a contractive projection in $C(K)$ onto $X$ iff $S \cap \sigma(S \cap D)=\varnothing$ and $X$ is isometric by restriction to $S \cup D$ to $Y=\{f$ in $C(S \cup D): f(x)=-f(\sigma(x))$ for all $x$ in $D\}$. Furthermore, if $X$ satisfies the above conditions, there is a one-to-one correspondence between the contractive projections onto $X$ and the continuous functions $c$ from $S \cup D$ into $[0,1]$ where $c(x)+c(\sigma(x))=1$ for $x$ in $D$ and $c(x)=1$ for $x$ in $S$. This correspondence is given as follows: For each contractive projection $P$ onto $X, \frac{1}{2}\left(P\left(1_{K}\right)(x)+1\right)$ is a continuous function which when restricted to $S \cup D$ has the desired properties; and for each function $c$ with the desired properties, there is a contractive projection, $P_{c}$, onto $X$ such that for $x$ in $S \cup D, c(x)=\frac{1}{2}\left(P_{c}\left(1_{K}\right)(x)+1\right)$ with $P_{c}=R^{-1} T_{c}$ where $R$ is the restriction isometry and $T_{c}$ is such that for all $f$ in $C(K), T_{c}(f)(x)=f(x)$ for $x$ in $S$ and $T_{c}(f)(x)=c(x) f(x)-(1-c(x)) f(\sigma(x))$ for $x$ in $D$.

Proof. That the conditions are necessary for the existence of a contractive projection onto $X$ is shown by Proposition 1.2 and Proposition 1.3. Proposition 2.2 shows these conditions are sufficient. We need only prove the statement about the one-to-one correspondence. By Proposition 1.2, for each contractive projection $P, c(x)=\frac{1}{2}\left(P\left(1_{K}\right)(x)+1\right)$ has the desired properties. For each $c$ with these properties, it is shown in the proof of Proposition 2.2 that $P_{c}=R^{-1} T_{c}$ is a contractive projection onto $X$. The 
proof is complete if we show that $c(x)=\frac{1}{2}\left(P_{c}\left(1_{K}\right)(x)+1\right)$ for all $x$ in $S \cup D$. But for $x$ in $S \cup D, P_{c}\left(1_{K}\right)(x)=R^{-1} T_{c}\left(1_{K}\right)(x)=T_{c}\left(1_{K}\right)(x)$; and $T_{c}\left(1_{K}\right)(x)=$ $c(x)-(1-c(x))=2 c(x)-1$ for $x$ in $D$ and $T_{c}\left(1_{K}\right)(x)=1=2 c(x)-1$ for $x$ in $S$. Hence, we have that for $x$ in $S \cup D, \frac{1}{2}\left(P_{c}\left(1_{K}\right)+1\right)=\frac{1}{2}(2 c(x)-1+1)=$ $c(x)$.

REMARKS. In [4] it is shown that if $X$ is a separating subspace of $C(K)$ with no double extreme points, there can be at most one contractive projection onto $X$; and furthermore there is a contractive projection onto $X$ iff $X$ is isometric by restriction to $S$, the closure of the single extreme points, to $C(S)$. This is a special case of Theorem 3.1.

\section{REFERENCES}

1. N. Dunford and J. T. Schwartz, Linear operators. I: General theory, Pure and Appl. Math., vol. 7, Interscience, New York, 1958. MR 22 \#8302.

2. J. Lindenstrauss, Extension of compact operators, Mem. Amer. Math. Soc. No. 48 (1964). MR 31 \#3828.

3. J. Lindenstrauss and D. Wulbert, On the classification of the Banach spaces whose duals are $L_{1}$ spaces, J. Functional Analysis 4 (1969), 332-349. MR 40 \#3274.

4. D. Wulbert, Some complemented function spaces in $C(X)$, Pacific J. Math. 24 (1968), 589-602. MR 36 \#6915.

Department of Mathematics, Wayne State University, Detroit, Michigan 48202 